\title{
An Invariant-Manifold Approach to Lumping
}

\author{
B.E. Okeke, M.R. Roussel* \\ Department of Chemistry and Biochemistry, University of Lethbridge \\ Lethbridge, Alberta T1K 3M4, Canada
}

\begin{abstract}
Differential equation models of chemical or biochemical systems usually display multiple, widely varying time scales, i.e. they are stiff. After the decay of transients, trajectories of these systems approach low-dimensional invariant manifolds on which the eventual attractor (an equilibrium point in a closed system) is approached, and in which this attractor is embedded. Computing one of these slow invariant manifolds (SIMs) results in a reduced model of dimension equal to the dimension of the SIM. Another approach to model reduction involves lumping, the formulation of a reduced set of variables that combine the original model variables and in terms of which the reduced model is framed. In this study, we combine lumping with a constructive method for SIMs based on the iterative solution of the invariance equation. We illustrate these methods using a simple model of a linear metabolic pathway, and a model for hydrogen oxidation. The former is treated with a linear lumping function, while a nonlinear lumping function based on a Lyapunov function is used in the latter.
\end{abstract}

Keywords and phrases: model reduction, slow invariant manifold, lumping, metabolic modeling, combustion modeling

Mathematics Subject Classification: 80A30, 92C45, 34C45

\section{Introduction}

Chemical and biochemical models can be very large. For example, Whitehouse and coworkers have studied a tropospheric chemistry model involving 3487 species [58]. An automatically generated model of Homo sapiens metabolism includes 3270 species [3]. When we add spatial dimensions, either to model coupled chemistry and transport, or to consider compartmentalization in living organisms, the number of variables to be included greatly increases. These very large models challenge even modern computers, particularly since the resulting differential equations are invariably stiff $[5,9,39]$, i.e. the ratio of the fastest to the slowest time scale captured by the equations is large. Modelers often say that they can test hypotheses faster than can be done in an experimental lab. This is certainly true for small models, but it is less obviously so for large, comprehensive models. This creates a dynamic tension in modeling between the study of small, efficient models, which necessarily leave out some details of the system under study, and large models which, if properly parameterized, allow for a more comprehensive study of a system but may require the resources of a large-scale computing center to tackle. Model reduction methods, which

* Corresponding author. E-mail: roussel@uleth.ca

(C) EDP Sciences, 2015 


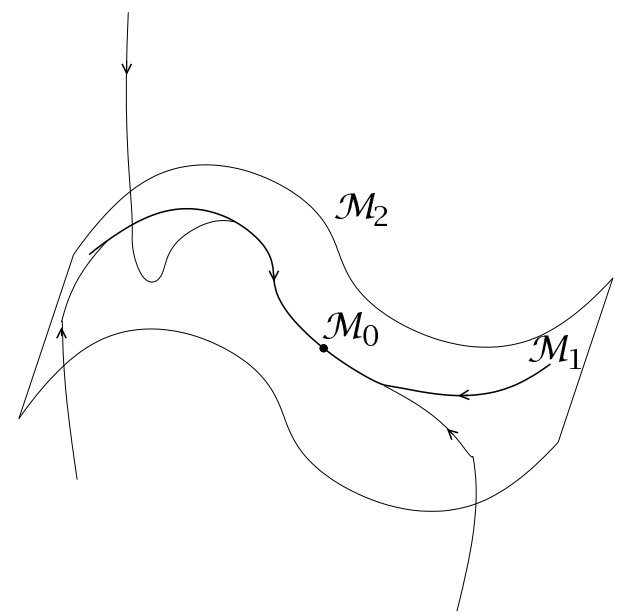

Figure 1. Schematic illustrating the flow geometry in phase space that allows for the construction of slow-time-scale models. Trajectories starting from anywhere in phase space are rapidly attracted to a set of embedded slow invariant manifolds of progressively lower dimensions. Here we show the flow first being attracted to a two-dimensional SIM $\left(\mathcal{M}_{2}\right)$, then to a one-dimensional SIM $\left(\mathcal{M}_{1}\right)$, and finally to the equilibrium point $\left(\mathcal{M}_{0}\right)$, itself an invariant manifold of the differential equations. Note that $\mathcal{M}_{1}$ here consists of two distinct trajectories entering $\mathcal{M}_{0}$ from opposite directions. Nevertheless, it can generally be computed as a single object.

are being actively studied by a number of groups around the world, allow for the study of larger models on relatively modest hardware.

There are a number of different ways to approach the model-reduction problem, reviewed elsewhere $[19,34,38,54]$. A theme that connects a number of methods is time-scale splitting [34]. Because of the large range of time scales in larger models, it is often possible to separate the motion into fast and slow modes. Typically, there are many fewer slow modes than fast modes, so that a slow-time-scale model is significantly reduced in size from the original model. Not only is the size of the model reduced, but the elimination of fast time scales also reduces the stiffness of the model, allowing for much more efficient integration of the remaining equations [33]. An accurate slow-time-scale model can simulate the behavior of the full model on these time scales, but of course the behavior on the fast time scales is lost. In some variations of time-scale splitting methods, notably in computational singular perturbation theory $[59,60]$ and in a new variation on the Fraser iterative method [55], the fast evolution can be obtained separately.

One approach to the construction of a slow-time-scale model is the explicit construction of a slow invariant manifold (SIM) $[10,13,15,17,35,43]$. In phase space, separation of time scales leads to the rapid approach of trajectories to low-dimensional invariant manifolds on which the slow evolution occurs (Fig. 1). The differential equations for the motion on a SIM constitutes a reduced model whose dimension is equal to that of the SIM. There is in fact a hierarchy of SIMs, each of which is embedded in the previous (higher-dimensional) member of the hierarchy [48,49], and reached after the exhaustion of transverse fast modes [27]. This hierarchy allows for the generation of different slow-time-scale models including more or fewer slow modes, as required by a particular application.

Lumping, also known as aggregation of variables, represents another line of attack to the model reduction problem. Here, we "lump together" or aggregate several variables of the original model to define the new variables of a reduced model [57]. Typically, the lumping function, the mapping from the original variables to the new variables, has been linear $[7,8,11,22,25,26,39,57]$, although nonlinear lumping functions are also possible [29-31]. In the classic formulation of the lumping problem, the goal is to find a lumping function $\zeta(\mathbf{s})$, with $\mathbf{s} \in \mathbb{R}^{N}$ the original variable vector, and $\zeta: \mathbb{R}^{N} \rightarrow \mathbb{R}^{d}, d \ll N$, such that 
there exists a closed evolution equation for the transformed variables $\mathbf{z}=\boldsymbol{\zeta}(\mathbf{s})$. We may seek conditions such that the system can be exactly $[30,57]$ or approximately lumped [22,26,31]. Additional properties of the lumped system may be sought, e.g. the representability of the lumped system by a chemical mechanism $[11,57]$.

In this paper, we turn this problem around. Rather than trying to find a lumping function $\zeta$ that leads to an accurate lumped model, we choose a lumping function that makes sense in the context of a given problem, and then determine the slow-time-scale rate equations for the lumped variables. This approach allows the modeler to choose aggregated variables that have a physical meaning, and brings together the SIM and lumping approaches to model reduction via the construction of a SIM parameterized by the lumped variables.

We note in passing that, whatever variables are used to parameterize the SIM, the qualitative picture presented in Fig. 1 implies the existence of a complementary set of variables for the fast fibers $[55,60]$. These variables represent modes that are exhausted during the fast relaxation $[27,28]$. In the simplest case, we would choose the fast variables so that they vanish on the SIM and therefore do not evolve on the slow time scale. This would represent a true slow/fast decomposition of the system variables. In practice, the "fast" variables are usually coupled to the slow variables once the SIM has been reached, i.e. they have a slow evolution [13]. A reduced model phrased exclusively in terms of the slow variables can still be obtained, but it in turn implies a coupled evolution of the fast variables. Equivalently, the variables in the original phase space, $\mathbf{s} \in \mathbb{R}^{N}$, can be described as being slaved to the variables used to describe the SIM on the slow time scale, $\mathbf{z} \in \mathbb{R}^{d}$. Thus, there exists a function $\mathbf{S}: \mathbb{R}^{d} \rightarrow \mathbb{R}^{N}$ mapping points on a SIM back to the original phase space and thus generating the slow-time-scale evolution in phase space. This function, along with the function $\zeta$ that defines the lumped variables, features prominently in the mathematical development to follow.

This paper is organized as follows: In Section 2, the necessary theory is developed. This includes the derivation of the invariance equation, whose solution space includes the slow invariant manifolds. In Section 3, the computation of the slow invariant manifolds of a linear metabolic pathway model is studied using linear lumping functions. Both one- and two-dimensional manifolds are constructed. Section 4 presents a study of a nonlinear lumping function in the context of a hydrogen oxidation model. This lumping function is a close relative of Lyapunov functions for chemical systems. We also review the use of iterative stabilization methods, which become necessary here. Finally, in Section 5, we provide some closing comments.

\section{Theory}

Our treatment here follows closely that of Goussis and Valorani [21].

\subsection{Time evolution of the lumped variables}

We assume that a modeler has chosen a lumping function $\boldsymbol{\zeta}$, which may be linear or nonlinear. This lumping function would normally be chosen based on physico-chemical considerations, as discussed in the examples of Sections 3 and 4 . Thus, the lumped variables are given by

$$
\mathbf{z}=\zeta(\mathbf{s})
$$

where $\mathbf{s} \in \mathbb{R}^{N}$ are the original model variables, and $\mathbf{z} \in \mathbb{R}^{d}$ are the lumped variables. Figure 2 illustrates the geometric meaning of a lumping function. For $d=1$, the case illustrated in Fig. 2, isosurfaces of $\zeta$ correspond to the set of $\mathbf{s}$ values that are lumped to the corresponding value of $z$. In higher dimensions, the set of states mapped onto any particular $\mathbf{z}$ is the intersection of the $d$ isosurfaces defined by equation (2.1).

We can differentiate equation (2.1) with respect to time to obtain an equation for the time evolution of the lumped variables:

$$
\dot{\mathrm{z}}=\zeta_{\mathrm{s}} \dot{\mathrm{s}}
$$




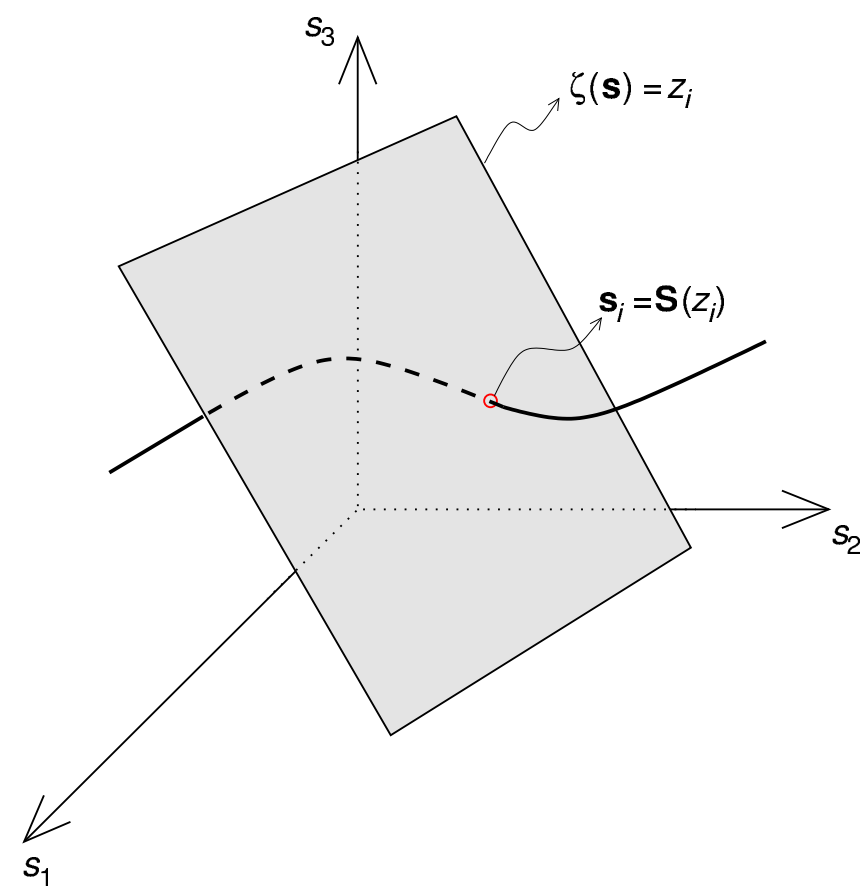

FiguRE 2. Diagram illustrating the geometric relationships between the original phase space, the lumping function $\zeta$, and the resulting parameterization of a one-dimensional slow manifold $\mathbf{S}(z)$. In practical calculations, we would distribute mesh points $z_{i}$ along the physically realizable region of the $z$ axis. Each value of $z=z_{i}$ corresponds to an isosurface of $\zeta(\mathbf{s})$. The slow manifold intersects the isosurface $\zeta(\mathbf{s})=z_{i}$ at a point $\mathbf{s}_{i}$, here illustrated as a small circle. The collection of these intersection points defines a map $\mathbf{S}: \mathbb{R}^{N} \rightarrow \mathbb{R}$ (for $d=1$ ) which defines a parameterization of the slow manifold by the lumped variable $z$.

where

$$
\zeta_{\mathbf{s}(i j)}=\frac{\partial \zeta_{i}}{\partial s_{j}}
$$

In the subsequent sections, we will show how to compute an equation for the slow invariant manifold parameterized by $\mathbf{z}$. Thus, given a $\mathbf{z}$, we will be able to compute a vector $\mathbf{s}$ lying in the SIM. If we represent this relationship by $\mathbf{s}=\mathbf{S}(\mathbf{z})$, then the evolution equation for the reduced model is

$$
\dot{\mathbf{z}}=\left[\boldsymbol{\zeta}_{\mathbf{s}} \dot{\mathbf{s}}\right]_{\mathbf{s}=\mathbf{S}(\mathbf{z})} .
$$

Note that, since $\boldsymbol{\zeta}(\mathbf{s})$ is a known lumping function, the matrix $\boldsymbol{\zeta}_{\mathbf{s}}$ is also known. The dependence of $\dot{\mathbf{s}}$ on $\mathbf{s}$ is of course known since this relation constitutes the evolution equation of the original model. The entire right-hand size of equation (2.2) is thus known except for the relationship between $\mathbf{s}$ and $\mathbf{z}$ on the manifold, i.e. the function $\mathbf{S}(\mathbf{z})$.

\subsection{The invariance equation}

A slow invariant manifold is a solution of the invariance equation, also known as the functional equation $[13,35]$. Here we derive a version of the invariance equation parameterized by arbitrary variables.

If the SIM is parameterized by the variables $\mathbf{z}$ then, as previously noted,

$$
\mathbf{s}=\mathbf{S}(\mathbf{z}) .
$$


Again, refer to Fig. 2. Let $\mathbf{z}_{i}$ be one of a set of points on some lattice in the physically realizable region of $\mathbb{R}^{d}$, where $i$ represents some indexing system on the lattice. The intersection of the SIM with the isosurface intersection $\boldsymbol{\zeta}(\mathbf{s})=\mathbf{z}_{i}$ occurs at a point in $\mathbb{R}^{N}$ denoted by $\mathbf{s}_{i}$. By computing these intersections for all $\mathbf{z}_{j}$ in a small neighborhood of $\mathbf{z}_{i}$, we can locally define the mapping (2.3), i.e. the parameterization of the SIM by $\mathbf{z}$, provided the SIM intersects $\zeta(\mathbf{s})=\mathbf{z}_{j}$ transversely everywhere in the specified neighborhood. A good lumping function will satisfy this transversality property everywhere within the physically realizable region of $\mathbb{R}^{N}$.

Differentiating equation (2.3) with respect to time, we obtain

$$
\dot{\mathbf{s}}=\mathbf{S}_{\mathrm{z}} \dot{\mathbf{z}}
$$

where

$$
\mathbf{S}_{\mathbf{z}(i j)}=\frac{\partial s_{i}}{\partial z_{j}}
$$

are a priori unknown partial derivatives giving the spatial rates of change of the $s_{i}$ with respect to the $z_{j}$ on the SIM. If we combine equation (2.4) with the evolution equation (2.2), we get

$$
\dot{\mathbf{s}}=\mathbf{S}_{\mathbf{z}}\left[\boldsymbol{\zeta}_{\mathbf{s}} \dot{\mathbf{s}}\right]_{\mathbf{s}=\mathbf{S}(\mathbf{z})},
$$

which can be rewritten as

$$
\left.\left(\mathbf{I}-\mathbf{S}_{\mathrm{z}} \boldsymbol{\zeta}_{\mathrm{s}}\right) \dot{\mathrm{s}}\right|_{\mathrm{s}=\mathrm{S}(\mathbf{z})}=\mathbf{0} .
$$

In this equation $\mathbf{I}$ is the $N \times N$ identity matrix. Equation (2.5) is a form of the invariance equation. This particular form of the invariance equation features prominently in the work of Alexander Gorban on model reduction $[16,18,20]$. A solution of the invariance equation is a function $\mathbf{S}(\mathbf{z})$ that satisfies (2.5). The original model provides an analytic form for $\dot{\mathbf{s}}(\mathbf{s})$. The choice of lumping function determines $\boldsymbol{\zeta}_{\mathbf{s}}(\mathbf{s})$. (For linear lumping, $\boldsymbol{\zeta}_{\mathbf{s}}$ is a constant matrix.) If $\mathbf{S}_{\mathbf{z}}(\mathbf{z})$ were known, then (2.5) would reduce to a set of algebraic equations for $\mathbf{s}$ for any given value of $\mathbf{z}$. However, $\mathbf{S}(\mathbf{z})$ itself is unknown, which of course makes its derivatives unknown as well. That being said, the observation that a knowledge of the value of $\mathbf{S}_{\mathbf{z}}$ would allow us to solve for $\mathbf{s}$ at the corresponding $\mathbf{z}$ leads to an iterative algorithm for $\mathbf{S}(\mathbf{z})$. This is the insight that led to the original iterative method for the solution of the invariance equation $[13,35]$.

Before we move on, note that not all of the $N$ components of equation (2.5) are independent [21]. Since the invariance equation derives from the lumping relationship (2.1), which provides $d$ equations, only $N-d$ of the components of the invariance equation are independent. To put it another way, we are only interested in solutions of (2.5) that are consistent with (2.1). There is no one correct way of combining the invariance equation with the lumping relation within an iterative scheme. We have explored a few options, which are described alongside the applications in the subsequent sections.

\section{The linear metabolic pathway model}

\subsection{Model equations}

Here we consider a model originally studied elsewhere [50] and recently revisited [37], namely a multistep metabolic transformation in which the product of one reaction is the reactant of the next, each step being catalyzed by a reversible Michaelian enzyme:

$$
\mathrm{S}_{\mathrm{i}-1} \underset{v_{i-}}{\stackrel{v_{i+}}{\rightleftharpoons}} \mathrm{S}_{\mathrm{i}}, \quad i=1,2, \ldots, N
$$

with

$$
\begin{aligned}
v_{i+} & =\frac{V_{i+}\left[\mathrm{S}_{i-1}\right] / K_{i+}}{1+\left[\mathrm{S}_{i-1}\right] / K_{i+}+\left[\mathrm{S}_{i}\right] / K_{i-}}, \\
v_{i-} & =\frac{V_{i-}\left[\mathrm{S}_{i}\right] / K_{i-}}{1+\left[\mathrm{S}_{i-1}\right] / K_{i+}+\left[\mathrm{S}_{i}\right] / K_{i-}},
\end{aligned}
$$


where $V_{i \pm}$ are the maximum velocities for each direction of an enzyme-catalyzed reaction step and $K_{i \pm}$ are the Michaelis constants of the appropriate enzyme-substrate complexes. This model has been found to be a challenging case study for model reduction, and is thus a stringent test for new methods.

Note that these equations describe an isomerization in a closed system. Accordingly, there is a mass conservation relation:

$$
\sum_{i=0}^{N}\left[\mathrm{~S}_{i}\right]=S_{T},
$$

where $S_{T}$ is the total amount of substrates in this linear pathway.

We rescale the variables and parameters as follows:

$$
\begin{array}{rlrl}
s_{i} & =\left[\mathrm{S}_{i}\right] / S_{T}, & & \\
\alpha_{i} & =K_{i+} / S_{T}, & & \beta_{i}=K_{i-} / S_{T}, \\
\gamma_{i} & =V_{i+} / K_{i+}, & \eta_{i}=V_{i-} / K_{i-} .
\end{array}
$$

This scaling differs slightly from that used in our previous work [50]. Because time was not rescaled, the equations we obtain are not fully dimensionless. However, this form is more symmetric than the equations we studied earlier, which has some advantages, particularly from the point of view of discussing how the relative time scales of various steps affect our ability to generate reduced models. After this scaling, we obtain the following rate equations:

$$
\begin{aligned}
\dot{s}_{0}= & \frac{\eta_{1} s_{1}}{1+s_{0} / \alpha_{1}+s_{1} / \beta_{1}}-\frac{\gamma_{1} s_{0}}{1+s_{0} / \alpha_{1}+s_{1} / \beta_{1}}, \\
\dot{s}_{i}= & \frac{\gamma_{i} s_{i-1}}{1+s_{i-1} / \alpha_{i}+s_{i} / \beta_{i}}-\frac{\eta_{i} s_{i}}{1+s_{i-1} / \alpha_{i}+s_{i} / \beta_{i}}-\frac{\gamma_{i+1} s_{i}}{1+s_{i} / \alpha_{i+1}+s_{i+1} / \beta_{i+1}}+\frac{\eta_{i+1} s_{i+1}}{1+s_{i} / \alpha_{i+1}+s_{i+1} / \beta_{i+1}}, \\
& \quad i=1,2, \ldots, N-1 .
\end{aligned}
$$

The system is closed by the dimensionless mass conservation relation:

$$
s_{N}=1-\sum_{i=0}^{N-1} s_{i} .
$$

For illustration, we specifically consider the case $N=5$ with the relative rates of the steps set as follows:

$$
\mathrm{S}_{0} \rightleftharpoons \mathrm{S}_{1} \stackrel{\text { slow }}{\rightleftharpoons} \mathrm{S}_{2} \stackrel{\text { slower }}{\rightleftharpoons} \mathrm{S}_{3} \rightleftharpoons \mathrm{S}_{4} \rightleftharpoons \mathrm{S}_{5} .
$$

Figure 3 shows some typical trajectories for this system along with the two-dimensional $\left(\mathcal{M}_{2}\right)$ and onedimensional $\left(\mathcal{M}_{1}\right)$ SIMs whose computation is described below. Note the hierarchical relaxation of trajectories, first to $\mathcal{M}_{2}$, then to $\mathcal{M}_{1}$ along which the equilibrium point $\left(\mathcal{M}_{0}\right)$ is approached. Computing $\mathcal{M}_{2}$ gives us a two-dimensional reduced model that contains both the motion on $\mathcal{M}_{2}$ towards $\mathcal{M}_{1}$ and the motion along $\mathcal{M}_{1}$ to the equilibrium point. Computing $\mathcal{M}_{1}$ gives us a one-dimensional reduced model for the approach to the equilibrium along this manifold. Depending on the range of timescales to be captured by a reduced model, either of these reductions could be useful.

\subsection{The one-dimensional manifold}

Given the assumed relative rates of the steps (3.3), a reasonable linear lumping function might be

$$
z=\zeta(\mathbf{s})=s_{0}+s_{1}+s_{2} .
$$

Note that equation (3.2) implies that physically meaningful values of $z$ lie in the range $0 \leq z \leq 1$. For this lumping function, we have

$$
\zeta_{\mathrm{s}}=\left[\begin{array}{lllll}
1 & 1 & 1 & 0 & 0
\end{array}\right] .
$$




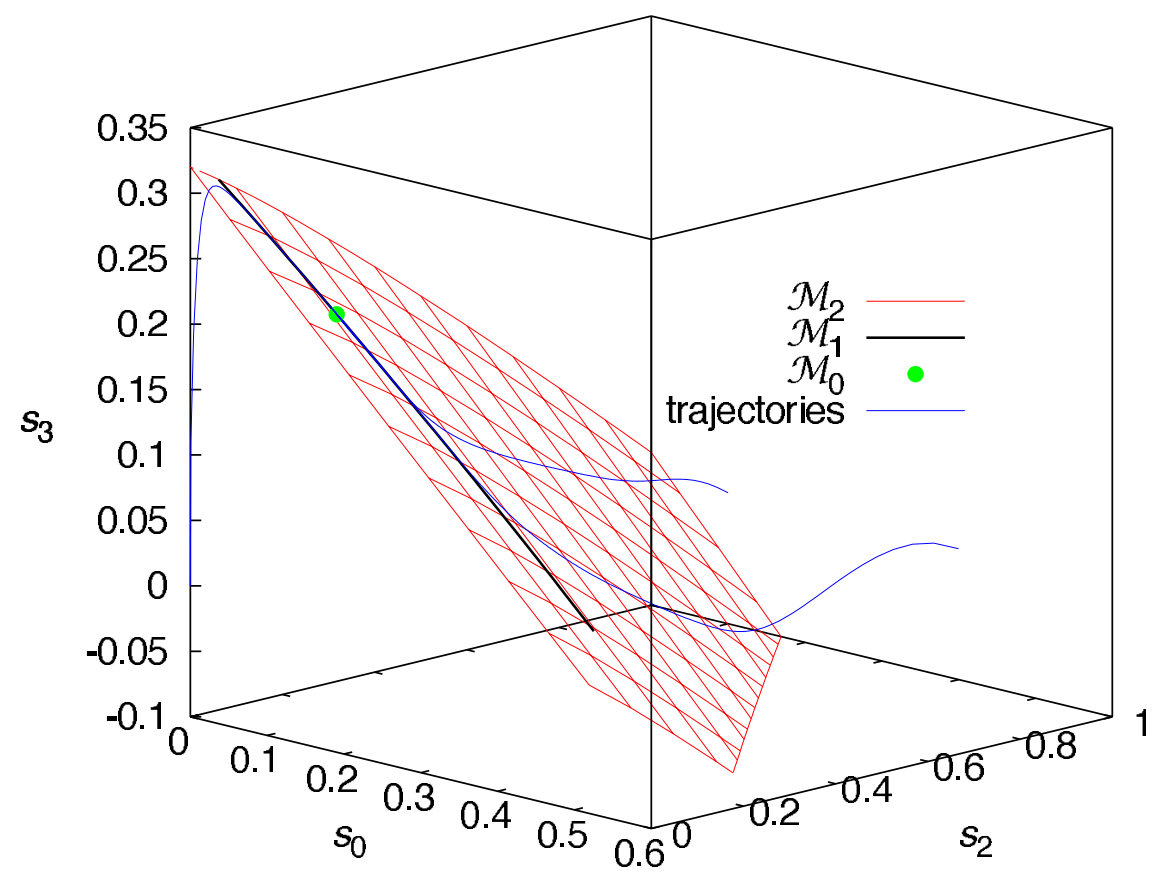

Figure 3. A few trajectories of the linear metabolic pathway plotted with the twodimensional $\left(\mathcal{M}_{2}\right)$ and one-dimensional $\left(\mathcal{M}_{1}\right)$ SIMs, as well as the equilibrium point $\left(\mathcal{M}_{0}\right)$. The parameters used to generate this figure are $\alpha_{i}=\beta_{i}=1 \forall i, \gamma_{1}=\eta_{1}=1 \mathrm{~s}^{-1}$, $\gamma_{2}=\eta_{2}=0.1 \mathrm{~s}^{-1}, \gamma_{3}=0.09 \mathrm{~s}^{-1}, \eta_{3}=0.05 \mathrm{~s}^{-1}, \gamma_{4}=\eta_{4}=\gamma_{5}=\eta_{5}=1 \mathrm{~s}^{-1}$.

There are only five entries in this matrix because $s_{5}$ is not an independent variable, being given by equation (3.2). $\mathbf{S}_{\mathbf{z}}$ is given by

$$
\mathbf{S}_{\mathbf{z}}=\left[\frac{\partial s_{0}}{\partial z} \frac{\partial s_{1}}{\partial z} \frac{\partial s_{2}}{\partial z} \frac{\partial s_{3}}{\partial z} \frac{\partial s_{4}}{\partial z}\right]^{\mathrm{T}}
$$

where the superscript $\mathrm{T}$ represents the matrix transpose. The invariance equation (2.5) reads

$$
\dot{s}_{i}-\frac{\partial s_{i}}{\partial z}\left(\dot{s}_{0}+\dot{s}_{1}+\dot{s}_{2}\right)=0, \quad i=0,1, \ldots 4
$$

However, only four of these five equations are independent given (3.4), which can be used to calculate, say, $s_{2}$ from $s_{0}$ and $s_{1}$ at any given $z$.

The iterative solution of the invariance equation involves the generation of an initial estimate of $\mathbf{S}_{\mathbf{z}}$, solving for $\mathbf{s}$ at each $\mathbf{z}$, which defines an approximation to $\mathbf{S}(\mathbf{z})$, then differentiating the latter approximation to refine the estimate of $\mathbf{S}_{\mathbf{z}}$. Because of the form of the rate functions in this model, it is not possible to carry out this algorithm analytically, as has been done in some other cases $[13,14,35,47$, 48]. Accordingly, we must proceed numerically, carrying out the calculation at discrete values of $z$ and estimating the derivatives from mesh data.

We denote the value of $s_{i}$ at mesh point $j$ in step $k$ of the iterative process by $s_{i, j}^{(k)}$. The estimate of $\partial s_{i} / \partial z$ at mesh point $j$ in step $k$ of the calculation is denoted by $\mathbf{S}_{\mathbf{z}(i, j)}^{(k)}$. In the example under consideration, equation (3.2) will be used to calculate $s_{5}$, and equation (3.4) will be used to calculate $s_{2}$. Define $\mathcal{I}$ as the ordered set of indices of the chemical species to be computed from the invariance equation (3.6), in this case $\{0,1,3,4\}$. The algorithm is as follows: 
1. Choose a number of mesh points, $M$. We assume here that mesh points are numbered 0 (corresponding to $z=0)$ to $M-1(z=1)$. Initialize an array of $z$ values at the $M$ mesh points chosen uniformly in the interval $[0,1]$.

2. Set $k=0$. Generate values for $s_{i, j}^{(0)}$ based on a some suitably chosen initial function.

3. Compute $\mathbf{S}_{\mathbf{z}}$ by central differences. At the edges, extrapolate the derivatives as follows:

$$
\begin{aligned}
& \left.\frac{\partial s_{i}}{\partial z}\right|_{z=0} \approx \mathbf{S}_{\mathbf{z}(i, 0)}^{(k)}=2 \mathbf{S}_{\mathbf{z}(i, 1)}^{(k)}-\mathbf{S}_{\mathbf{z}(i, 2)}^{(k)}, \\
& \left.\frac{\partial s_{i}}{\partial z}\right|_{z=1} \approx \mathbf{S}_{\mathbf{z}(i, M-1)}^{(k)}=2 \mathbf{S}_{\mathbf{z}(i, M-2)}^{(k)}-\mathbf{S}_{\mathbf{z}(i, M-3)}^{(k)}
\end{aligned}
$$

Aside: There are no imposed boundary conditions in this method. The entire manifold, including points at the boundary of the computational domain, is generated by the iterative process from whatever initial function is used. In numerical implementations, we sometimes observe iterative instabilities that travel inward from the boundaries. These instabilities often set in as we approach either a bifurcation that destroys a SIM, or a region of parameter space in which the SIM is not well parameterized by the chosen $\mathbf{z}$. The instabilities are presumably due to the introduction of discontinuities in higher derivatives introduced by the change in differencing formulas that is necessary at the boundaries. The nearer the SIM is to a bifurcation or other major change in geometry, the less stable the iterative process becomes and the more likely it is that nonsmoothness of the approximated derivatives will create ripples at the boundary leading to destabilization. Empirically, we have found that estimating the derivatives by extrapolation at the boundaries, which results in some smoothing at the edges, is superior to the use of one-sided differences in the sense that it allows convergence of the iterations over a broader parameter range. Of course, it is possible that using more sophisticated means of estimating the derivatives would have a similar effect, the use of central differences being, admittedly, among the simplest possible methods.

4. For each $i \in \mathcal{I}$ in turn, solve for $s_{i, j}^{(k+1)}$ from the invariance equation (3.6) at each mesh point using the derivative estimates $\mathbf{S}_{\mathbf{z}(i, j)}^{(k)}$ and the function values $s_{i^{\prime}, j}^{(k+1)}$ for $i^{\prime}<i, s_{i^{\prime}, j}^{(k)}$ for $i^{\prime}>i$. We have referred to this procedure elsewhere as chained iteration [49], and it tends to accelerate convergence. The numerical (e.g. Newton) solver used to find solutions of the invariance equation requires a seed. It is advantageous to compute the $s_{i, j}^{(k+1)}$ working outwards from the equilibrium point, using the newly computed neighboring value as the seed for the solver.

5. Define the iterative error

$$
E_{k+1}=\max _{i, j}\left|s_{i, j}^{(k+1)}-s_{i, j}^{(k)}\right| .
$$

If $E_{k+1}>\epsilon$, where $\epsilon$ is a pre-selected tolerance, set $k=k+1$ and go to step 3. Otherwise, terminate.

Step 2 requires an initial function. We have found that quasi-equilibrium initial functions are particularly effective. To compute these functions, we assume that the components of the lump defined by equation (3.4) are mutually equilibrated with total value $z$, i.e. that $v_{1+}=v_{1-}$ and $v_{2+}=v_{2-}$ holds locally at each $z$. For the components that do not belong to the lump, note that equations (3.2) and (3.4) together imply that

$$
s_{3}+s_{4}+s_{5}=1-z .
$$

We again assume that these values are mutually equilibrated, i.e. that $v_{4+}=v_{4-}$ and $v_{5+}=v_{5-}$ locally at each $z$. The resulting quasi-equilibrium expressions are as follows:

$$
\begin{aligned}
& s_{0}^{(0)}=\frac{\eta_{1} \eta_{2} z}{\eta_{1} \eta_{2}+\gamma_{1} \eta_{2}+\gamma_{1} \gamma_{2}}, \\
& s_{1}^{(0)}=\frac{\gamma_{1} \eta_{2} z}{\eta_{1} \eta_{2}+\gamma_{1} \eta_{2}+\gamma_{1} \gamma_{2}}, \\
& s_{2}^{(0)}=\frac{\gamma_{1} \gamma_{2} z}{\eta_{1} \eta_{2}+\gamma_{1} \eta_{2}+\gamma_{1} \gamma_{2}},
\end{aligned}
$$




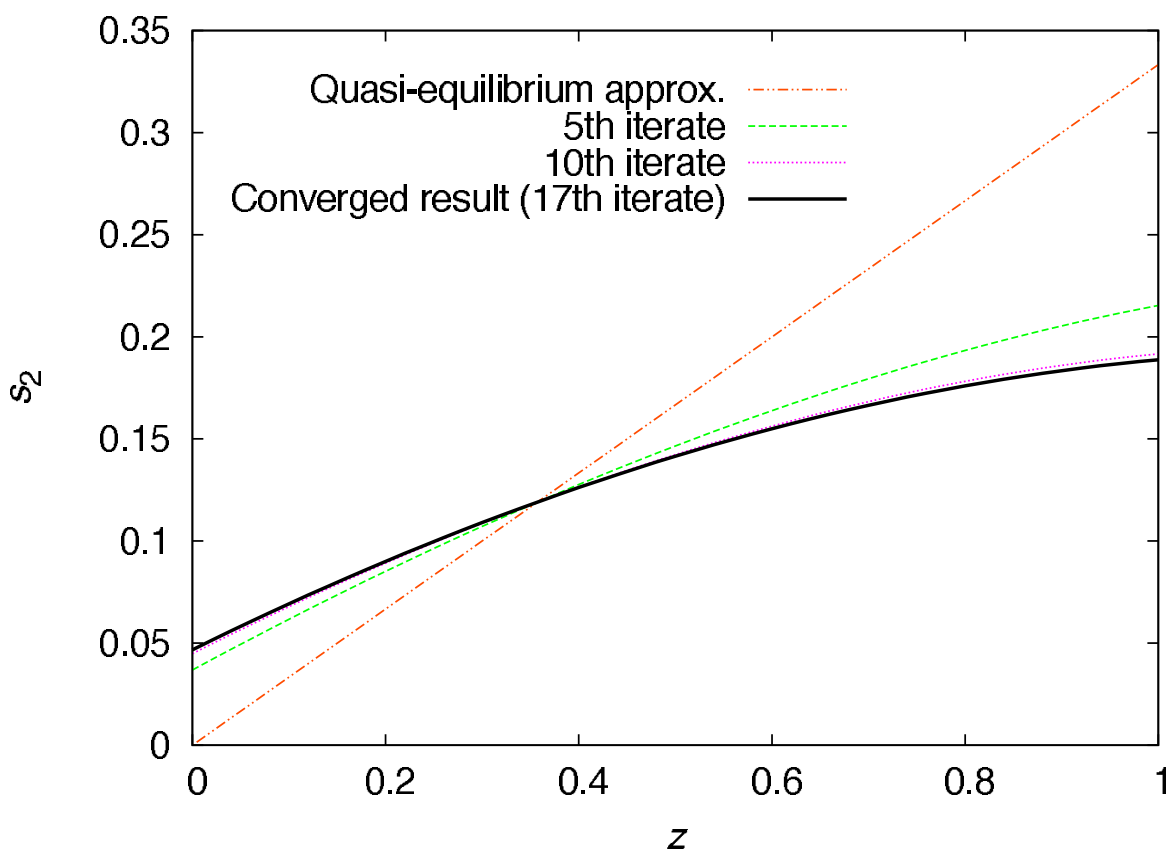

FiguRE 4 . Iterates of the $s_{2}$ component of the one-dimensional manifold for the same parameters as in Fig. 3, computed computed with $M=51$ mesh points and tolerance $\epsilon=10^{-4}$. The converged manifold is replotted as $\mathcal{M}_{1}$ in Fig. 3.

$$
\begin{aligned}
& s_{3}^{(0)}=\frac{\eta_{4} \eta_{5}(1-z)}{\eta_{4} \eta_{5}+\gamma_{4} \eta_{5}+\gamma_{4} \gamma_{5}}, \\
& s_{4}^{(0)}=\frac{\gamma_{4} \eta_{5}(1-z)}{\eta_{4} \eta_{5}+\gamma_{4} \eta_{5}+\gamma_{4} \gamma_{5}}, \\
& s_{5}^{(0)}=\frac{\gamma_{4} \gamma_{5}(1-z)}{\eta_{4} \eta_{5}+\gamma_{4} \eta_{5}+\gamma_{4} \gamma_{5}} .
\end{aligned}
$$

Figure 4 demonstrates the convergence of the iterative scheme. For parameters that fit the physical situation represented by the lumping function chosen, i.e. those in which the first three components are separated from the remaining components of the pathway by a slow step, convergence tends to be rapid, as illustrated here. Note that the difference between $\left(\gamma_{2}, \eta_{2}\right)$ and $\left(\gamma_{3}, \eta_{3}\right)$ need not be large, as demonstrated by this example. In these cases, the SIM can be significantly different in shape than the quasi-equilibrium initial function without preventing the basic scheme from converging. On the other hand, in parameter regimes where the lumping function is inappropriate, the iterations do not converge. Of course, to carry out these computations, we need kinetic parameters, so we would normally know in advance (or could determine dynamically) what steps are fast or slow.

Equation (2.2) gives the time evolution of the reduced model. For this particular model, from equation (3.4), we have

$$
\dot{z}=\dot{s}_{0}(\mathbf{s}(z))+\dot{s}_{1}(\mathbf{s}(z))+\dot{s}_{2}(\mathbf{s}(z)) .
$$

We can check how precisely the manifold has been computed by integrating both the full model and the reduced model starting from corresponding initial conditions, i.e. choosing the initial conditions on the SIM corresponding to the initial value of $z$ used in the reduced model. To integrate the reduced model, we need values of $\mathbf{s}$ at arbitrary values of $z$. We obtain these values by cubic spline interpolation on the computed SIM. Figure 5 shows trajectories of the full model, of the model reduced to our best approximation to $\mathcal{M}_{1}$, and of the model reduced to the quasi-equilibrium approximation (QEA) manifold 


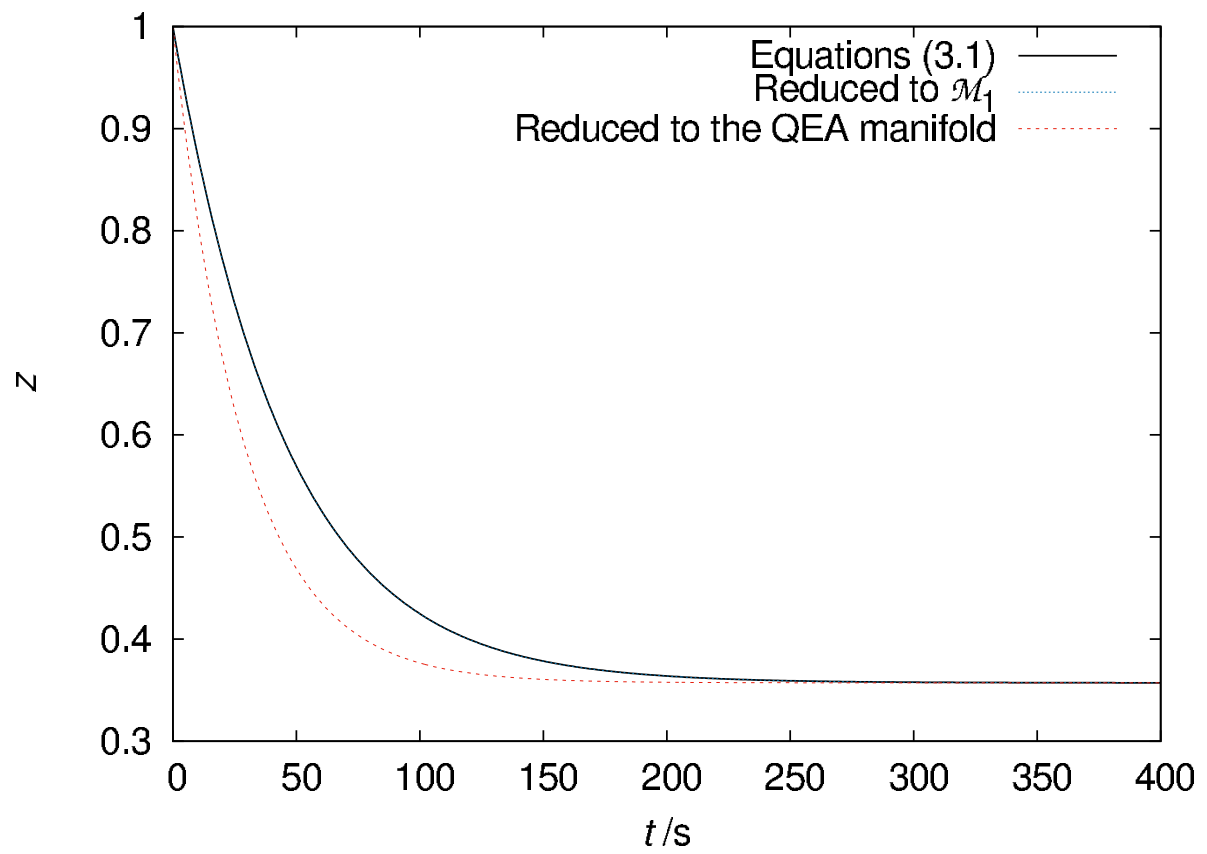

Figure 5. Time courses for the full model [equations (3.1)], for the model reduced to our best approximation to $\mathcal{M}_{1}$ (indistinguishable from the full model on the scale of this figure), and for the model reduced to the QEA. All parameters are as in Fig. 3. The initial conditions are described in the text.

that was used to initialize the iterative process, equation (3.8). The two reduced models were integrated from the initial condition $z=1$. The exact model was integrated from $\mathbf{s}=\mathbf{S}(1)$. On the scale of this figure, the exact solution and the solution for the model reduced to $\mathcal{M}_{1}$ are indistinguishable, indicating that we have accurately approximated the latter. On the other hand, the model reduced to the QEA manifold decays to equilibrium much more rapidly than either of the other two.

A signed relative error can be calculated at each time point by

$$
\sigma(t)=\left[z_{\text {full }}(t)-z_{\text {reduced }}(t)\right] / z_{\text {full }}(t) .
$$

Figure 6 shows these errors. In this case, we started the full model from the corresponding point on each of the manifolds, i.e. the error for our computed $\mathcal{M}_{1}$ was computed from a reference trajectory started on $\mathcal{M}_{1}$, while the reference trajectory used to compute the error in the QEA was started from a point on the QEA, in each case at $z=1$. The initial increase in the error in both cases is due to the non-invariance of the manifolds. However, the much smaller value of the error for our computed $\mathcal{M}_{1}$ indicates that this manifold is much closer to invariance than the QEA, for which the error briefly exceeds $10 \%$. Moreover, the error in the model reduced to $\mathcal{M}_{1}$ could be further decreased by computing this manifold with a tighter tolerance $\epsilon$.

\subsection{The two-dimensional manifold}

In this section, we continue our analysis of model (3.3), this time computing a two-dimensional SIM. Physically, the following seems like an obvious lumping function as it groups together species that equilibrate rapidly relative to the two slow steps:

$$
\left[\begin{array}{l}
z_{1} \\
z_{2}
\end{array}\right]=\boldsymbol{\zeta}(\mathbf{s})=\left[\begin{array}{c}
s_{0}+s_{1} \\
s_{2}
\end{array}\right] .
$$




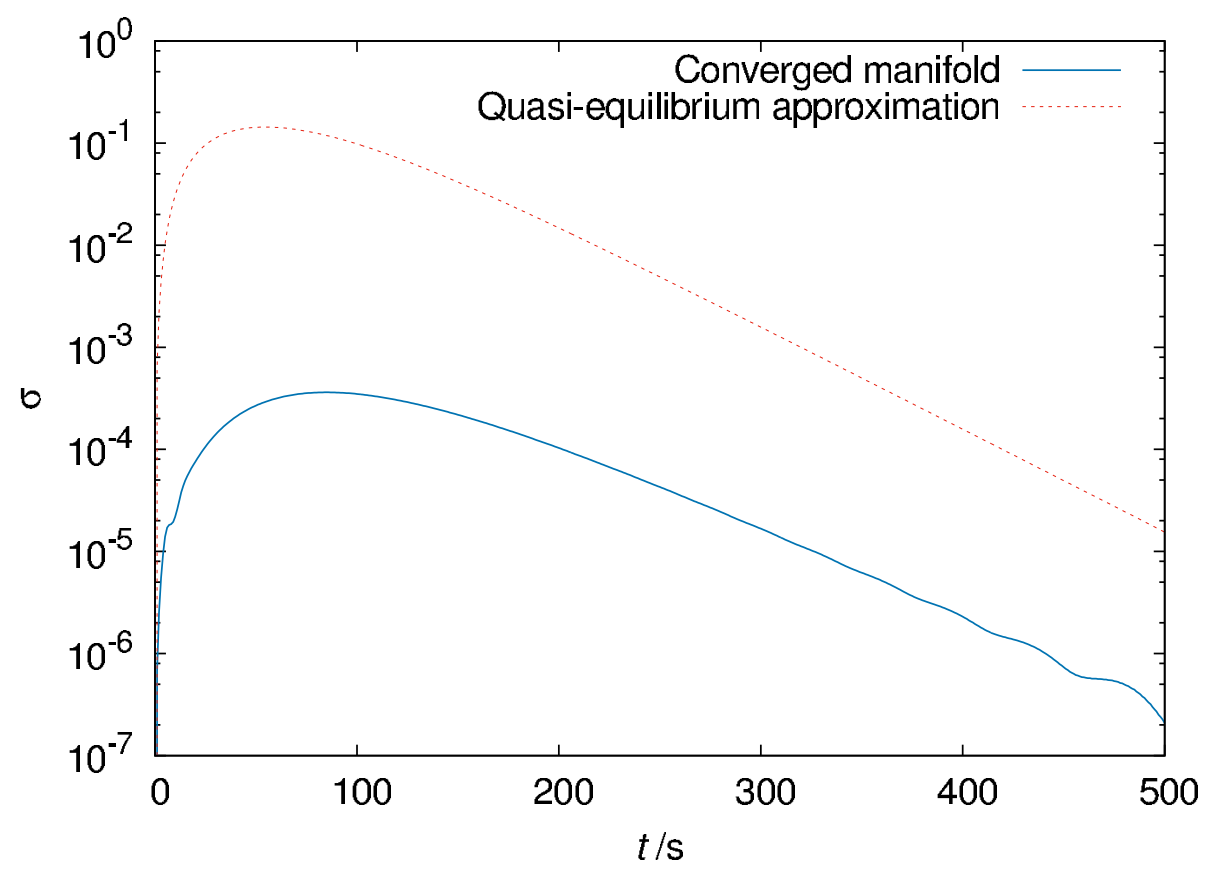

FiguRE 6. Relative errors of the models reduced to our best approximation to $\mathcal{M}_{1}$ or to the QEA along trajectories started from $z=1$. All parameters are as in Fig. 3.

The SIM now satisfies equations (3.2), (3.11) (solved for, say, $s_{1}$ and $s_{2}$ in terms of $z_{1}$ and $z_{2}$ ), as well as the invariance equation components

$$
\begin{aligned}
& \left(1-\frac{\partial s_{0}}{\partial z_{1}}\right) \dot{s}_{0}-\frac{\partial s_{0}}{\partial z_{1}} \dot{s}_{1}-\frac{\partial s_{0}}{\partial z_{2}} \dot{s}_{1}=0 \\
& -\frac{\partial s_{3}}{\partial z_{1}} \dot{s}_{0}-\frac{\partial s_{3}}{\partial z_{1}} \dot{s}_{1}-\frac{\partial s_{3}}{\partial z_{2}} \dot{s}_{2}+\dot{s}_{3}=0 \\
& -\frac{\partial s_{4}}{\partial z_{1}} \dot{s}_{0}-\frac{\partial s_{4}}{\partial z_{1}} \dot{s}_{1}-\frac{\partial s_{4}}{\partial z_{2}} \dot{s}_{2}+\dot{s}_{4}=0
\end{aligned}
$$

The computation is in all important respects essentially identical to that of the one-dimensional SIM, including the method for constructing a quasi-equilibrium initial function.

Figure 7 shows the $s_{3}$ component of both the initial quasi-equilibrium approximation along with the converged manifold, this time obtained in five iterates. This extremely fast convergence is due to the large separation of time scales between the two slowest modes and the next slowest mode.

Finally, we show the trajectories of the model reduced to $\mathcal{M}_{2}$ (Fig. 8). Trajectories of the full model started on $\mathcal{M}_{2}$ (not shown) are indistinguishable from these on the scale of this figure. As noted previously, lower-dimensional members of the relaxation hierarchy are embedded in higher-dimensional members. Here, we clearly see the trajectories on $\mathcal{M}_{2}$ approaching the one-dimensional SIM, $\mathcal{M}_{1}$, on the way to the equilibrium point, $\mathcal{M}_{0}$.

\section{A hydrogen oxidation model}

In the previous section, we considered straightforward linear lumping functions. Here, we demonstrate that the method can be adapted to nonlinear lumping functions. We consider a hydrogen oxidation model 


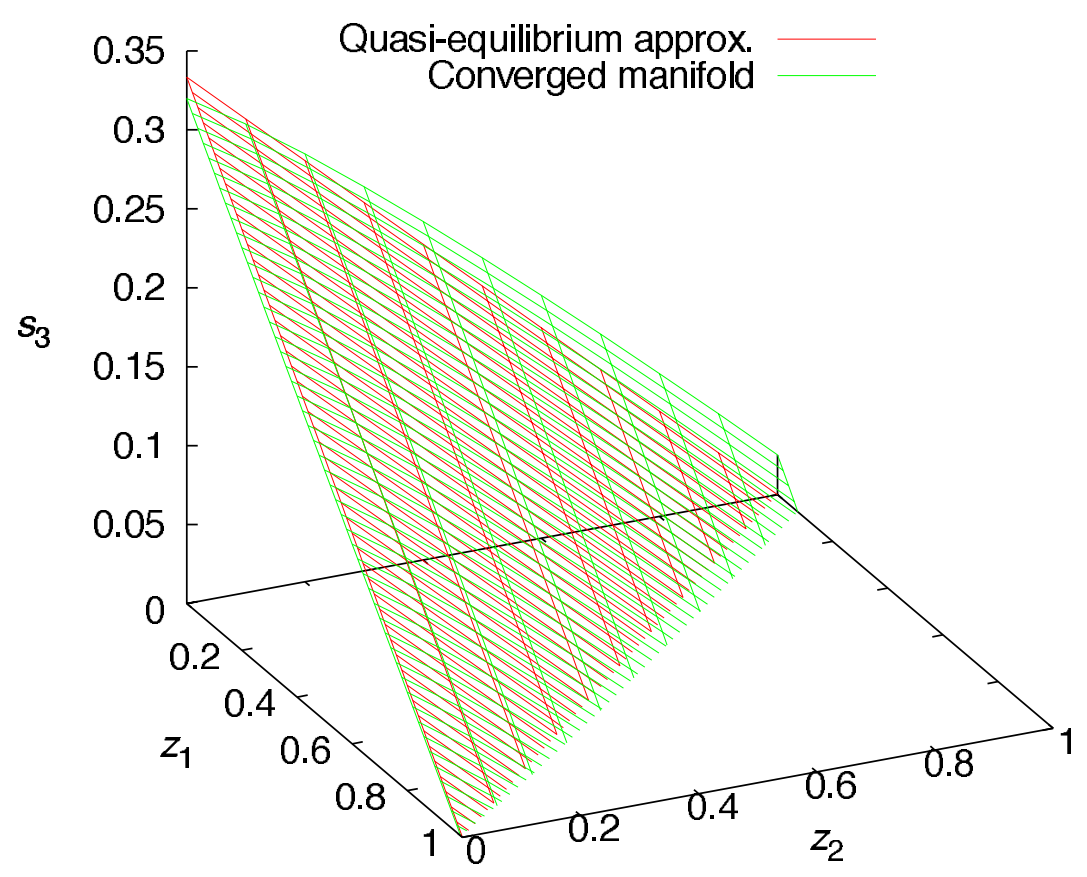

FIGURE $7 . s_{3}$ components of the quasi-equilibrium initial function and converged twodimensional SIM (5th iterate) for the linear metabolic pathway model. All parameters were set as in Fig. 3. The computation was carried out on a $51 \times 51$ grid with a tolerance of $\epsilon=10^{-4}$. The converged result is replotted as $\mathcal{M}_{2}$ in Fig. 3 .

previously studied by Gorban and coworkers [20]:

$$
\begin{aligned}
& \mathrm{H}_{2} \underset{k_{1}^{-}}{\stackrel{k_{1}^{+}}{\rightleftharpoons}} 2 \mathrm{H} \\
& \mathrm{O}_{2} \underset{k_{2}^{-}}{\stackrel{k_{2}^{+}}{\rightleftharpoons}} 2 \mathrm{O} \\
& \mathrm{H}_{2} \mathrm{O} \underset{k_{3}^{-}}{\stackrel{k_{3}^{+}}{\rightleftharpoons}} \mathrm{H}+\mathrm{OH} \\
& \mathrm{H}_{2}+\mathrm{O} \underset{k_{4}^{-}}{\stackrel{k_{4}^{+}}{\rightleftharpoons}} \mathrm{H}+\mathrm{OH} \\
& \mathrm{O}_{2}+\mathrm{H} \underset{k_{5}^{-}}{\stackrel{k_{5}^{+}}{\rightleftharpoons}} \mathrm{O}+\mathrm{OH} \\
& \mathrm{H}_{2}+\mathrm{O} \underset{k_{6}^{-}}{\stackrel{k_{6}^{+}}{\rightleftharpoons}} \mathrm{H}_{2} \mathrm{O} \text {. }
\end{aligned}
$$

This is a small model, with six chemical species and two mass conservation relations:

$$
\begin{aligned}
& m_{\mathrm{H}}=2\left[\mathrm{H}_{2}\right]+2\left[\mathrm{H}_{2} \mathrm{O}\right]+[\mathrm{H}]+[\mathrm{OH}], \\
& m_{\mathrm{O}}=2\left[\mathrm{O}_{2}\right]+\left[\mathrm{H}_{2} \mathrm{O}\right]+[\mathrm{O}]+[\mathrm{OH}] .
\end{aligned}
$$

There are thus just four independent concentrations, which we take to be $\left[\mathrm{H}_{2}\right],\left[\mathrm{O}_{2}\right],\left[\mathrm{H}_{2} \mathrm{O}\right]$ and $[\mathrm{OH}]$. Despite its small size, this model is useful for testing ideas about model reduction as it has both oneand two-dimensional SIMs [20]. 


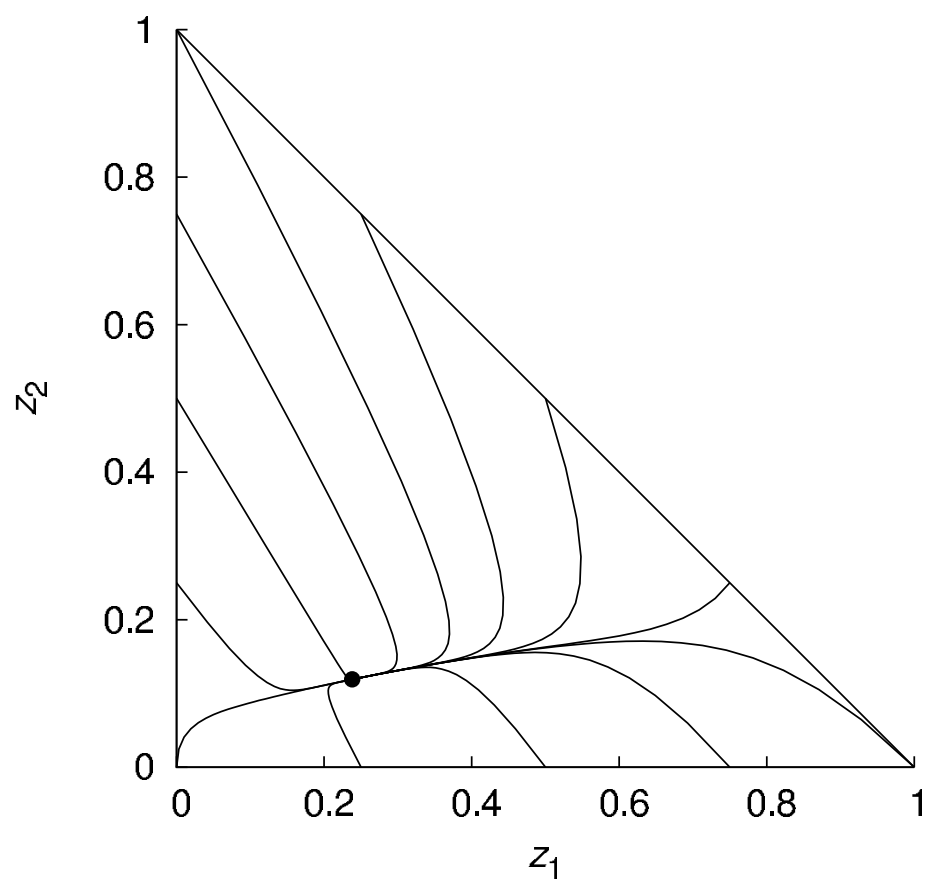

Figure 8. Trajectories of the reduced model on $\mathcal{M}_{2}$. All parameters are as in Fig. 3. The solid dot marks the equilibrium point. The triangular prism within which the trajectories are plotted is the physically realizable portion of the reduced phase space in which $z_{1}+z_{2} \leq 1$.

A lumping function can be any quantity such that each $\mathbf{z}=\boldsymbol{\zeta}(\mathbf{s})$ uniquely specifies an $\mathbf{s}$ along the slow manifold. In other words, $\mathbf{S}(\mathbf{z})$ must be a single-valued function. For the hydrogen oxidation model considered here, one possibility is the "partial" Lyapunov function

$$
\zeta(\mathbf{s})=\left[\mathrm{H}_{2}\right]\left\{\ln \left(\frac{\left[\mathrm{H}_{2}\right]}{\left[\mathrm{H}_{2}\right]_{\mathrm{eq}}}\right)-1\right\}+\left[\mathrm{O}_{2}\right]\left\{\ln \left(\frac{\left[\mathrm{O}_{2}\right]}{\left[\mathrm{O}_{2}\right]_{\mathrm{eq}}}\right)-1\right\}+\left[\mathrm{H}_{2} \mathrm{O}\right]\left\{\ln \left(\frac{\left[\mathrm{H}_{2} \mathrm{O}\right]}{\left[\mathrm{H}_{2} \mathrm{O}\right]_{\mathrm{eq}}}\right)-1\right\},
$$

where the subscript 'eq' indicates equilibrium concentrations. Note the analogy to the conventional Lyapunov function for closed, isothermal chemical systems, in which we would sum over all species [51] rather than just retaining terms for the three stable species, the reactants and products of the reaction. In other words, this is the Lyapunov-type function we would write for this chemical reaction if we did not know the mechanism, i.e. if we had no idea what intermediates the system had. Since the thermodynamic driving force to equilibrium is independent of mechanism, this function should decrease monotonically to its minimum at the equilibrium point along the SIM, making it a suitable nonlinear lumping function.

We order the variables lexically, i.e.

$$
\mathbf{s}=\left[\left[\mathrm{H}_{2}\right]\left[\mathrm{H}_{2} \mathrm{O}\right][\mathrm{OH}]\left[\mathrm{O}_{2}\right]\right] .
$$

For the choice of $\zeta(4.2), \zeta_{\mathbf{s}}$ has a simple form:

$$
\zeta_{\mathbf{s}}=\left[\ln \left(\frac{\left[\mathrm{H}_{2}\right]}{\left[\mathrm{H}_{2}\right]_{\mathrm{eq}}}\right) \ln \left(\frac{\left[\mathrm{H}_{2} \mathrm{O}\right]}{\left[\mathrm{H}_{2} \mathrm{O}\right]_{\mathrm{eq}}}\right) \quad 0 \quad \ln \left(\frac{\left[\mathrm{O}_{2}\right]}{\left[\mathrm{O}_{2}\right]_{\mathrm{eq}}}\right)\right] .
$$

From this point, we construct the invariance equation (2.5) as we have done previously. We get

$$
\dot{s}_{i}-\frac{\partial s_{i}}{\partial z} \sum_{j \in\{1,2,4\}} \dot{s}_{j} \ln \left(\frac{s_{j}}{s_{j, \text { eq }}}\right)=0 .
$$


We need to solve three components of equation (4.3) along with $z=\zeta(\mathrm{s})$, with $\zeta(\mathrm{s})$ given by equation (4.2), and using the mass conservation relations (4.1) to compute $[\mathrm{H}]$ and $[\mathrm{O}]$ as needed. Given the nonlinearity in (4.2), this equation cannot be used straightforwardly to eliminate one of the variables, as was done with the linear lumping functions in Section 3. Accordingly, we treat the three independent components of equation (4.3) along with (4.2) as a system of equations to be solved at each $z$.

Iterations based directly on the above equations diverge. It is therefore necessary to apply a stabilization method $[10,45,50]$. We review the basic ideas of iterative stabilization here.

We can think of the invariance equation as a functional relationship between the manifold coordinates and the derivatives of these coordinates, in this case with respect to the parameterizing variable $z$, a viewpoint that was particularly emphasized by Fraser [13]:

$$
\mathbf{s}=\mathcal{F}\left(\mathbf{S}_{\mathbf{z}}, \mathbf{z}\right) .
$$

The functional $\mathcal{F}\left(\mathbf{S}_{\mathbf{z}}, \mathbf{z}\right)$ is, in many cases, including the one considered in this section, implicitly defined by the invariance equation rather than explicitly defined. In the current formalism, we must think of the invariance equation itself as being augmented by the lumping relationship. Suppose that we have solved the invariance and lumping equations using some given $\mathbf{S}_{\mathbf{z}}$ at a particular $\mathbf{z}$. Thus we have an $\mathbf{s}$ that satisfies (4.4) locally for the given $\mathbf{S}_{\mathbf{z}}$. Add $w \mathbf{s}$ to both sides of equation (4.4), where $w$ is an arbitrary weight, and rearrange to

$$
\mathbf{s}=\frac{\mathcal{F}\left(\mathbf{S}_{\mathbf{z}}, \mathbf{z}\right)+w \mathbf{s}}{1+w}
$$

We can convert this relationship, which must be identically satisfied for any $\mathbf{s}$ that solves (4.4) and for any $w$, to an iteration by labeling as follows:

$$
\mathbf{s}^{(k+1)}=\frac{\mathcal{F}\left(\mathbf{S}_{\mathbf{z}}^{(k)}, \mathbf{z}\right)+w \mathbf{s}^{(k)}}{1+w} .
$$

For some types of instabilities, iteration can be stabilized for sufficiently large values of $w[10,45]$. The basic idea is that we are slowing convergence by mixing in some amount of the previous iterate, but are trading this slower convergence for improved stability.

Because stabilization slows convergence, roughly by a factor of $1+w$, the differences between iterates will necessarily be smaller by a similar factor. In order to reach the same accuracy, we must therefore adjust our stopping criterion by reducing our tolerance by a factor of $(1+w)^{-1}$ relative to what we would have been willing to accept in simple iterations.

We apply this stabilization method to the current problem using the parameters given by Gorban and coworkers [20], namely

$$
\begin{gathered}
k_{1}^{+}=2, k_{2}^{+}=k_{3}^{+}=1, k_{4}^{+}=k_{5}^{+}=1000, k_{6}^{+}=100, \\
{\left[\mathrm{H}_{2}\right]_{\mathrm{eq}}=0.27,\left[\mathrm{H}_{2} \mathrm{O}\right]_{\mathrm{eq}}=0.7,[\mathrm{OH}]_{\mathrm{eq}}=0.01,\left[\mathrm{O}_{2}\right]_{\mathrm{eq}}=0.135,[\mathrm{H}]_{\mathrm{eq}}=0.05,[\mathrm{O}]_{\mathrm{eq}}=0.02 .}
\end{gathered}
$$

The reverse rate constants $k_{i}^{-}$are recovered by applying the principle of detailed balance to each elementary reaction in the mechanism. The initial function is, again, a quasi-equilibrium function. This time, we assume that the last three reactions are in quasi-equilibrium given their large forward rate constants. Solving these three equilibrium relationships along with $z=\zeta(\mathbf{s})$ and the mass conservation relations gives us a physically sensible initial function from which to start iteration. The resulting stabilized iterative behavior is shown in Fig. 9. Convergence is considerably slowed, as expected. Nevertheless, the calculation is readily managed on an ordinary desktop computer.

We can again study the relative error (3.10) along a trajectory. Due to the singularity at the equilibrium point for this particular lumping function, we cannot compute the SIM right through this point. However, we can add this point to the computed data set after the computation since we know its coordinates. Using piecewise cubic interpolation in the computation of $\dot{z}$ then gives very good results, as seen in Fig. 10, with the relative error never exceeding $1.2 \%$. The sharp features at small $t$ are due to small errors in the construction of the manifold, which result in a rapid transient motion about the computed manifold in the full model before this trajectory starts shadowing the approximate manifold. 


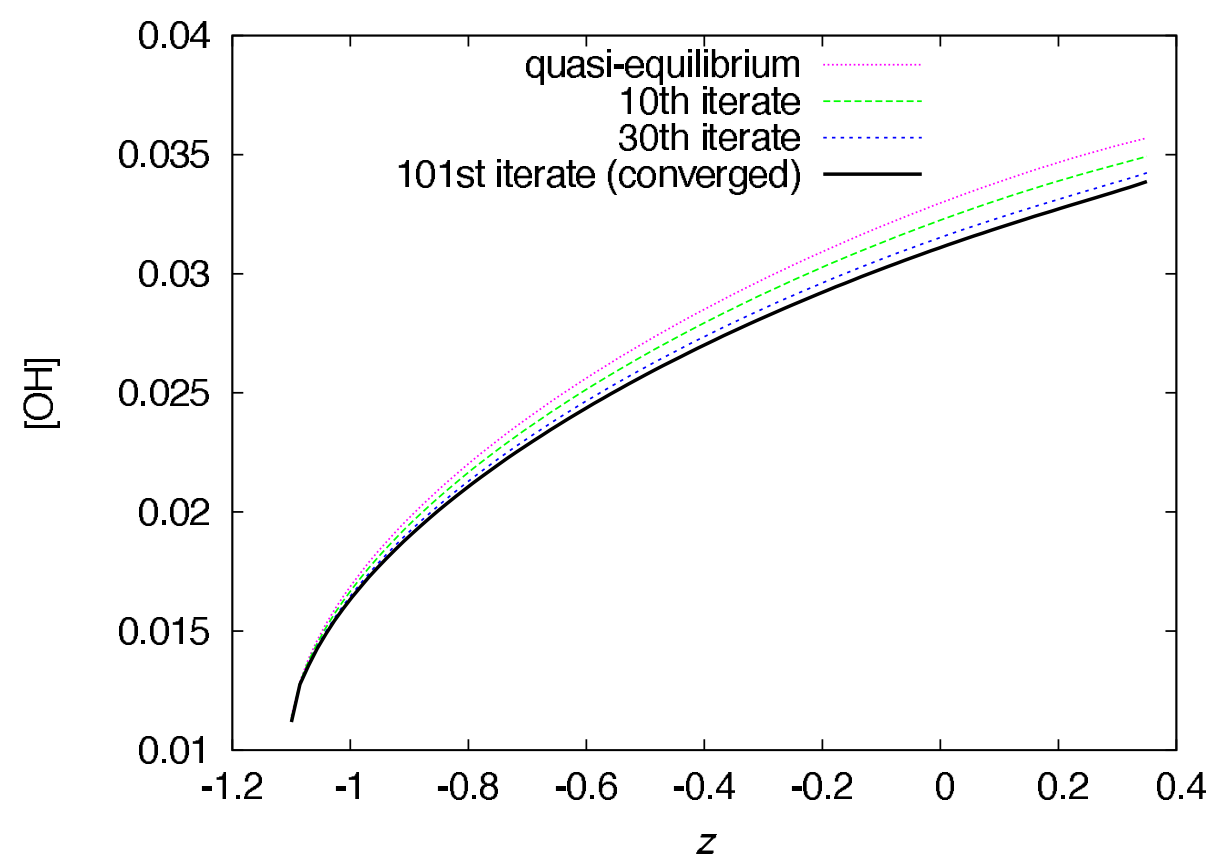

FiguRE 9. $[\mathrm{OH}]$ component of the iterates for the stabilized iteration method applied to the $\mathrm{H}_{2}$ oxidation mechanism with lumping function (4.2), $w=20$, tolerance $10^{-4} /(1+w)$, and using 100 mesh points.

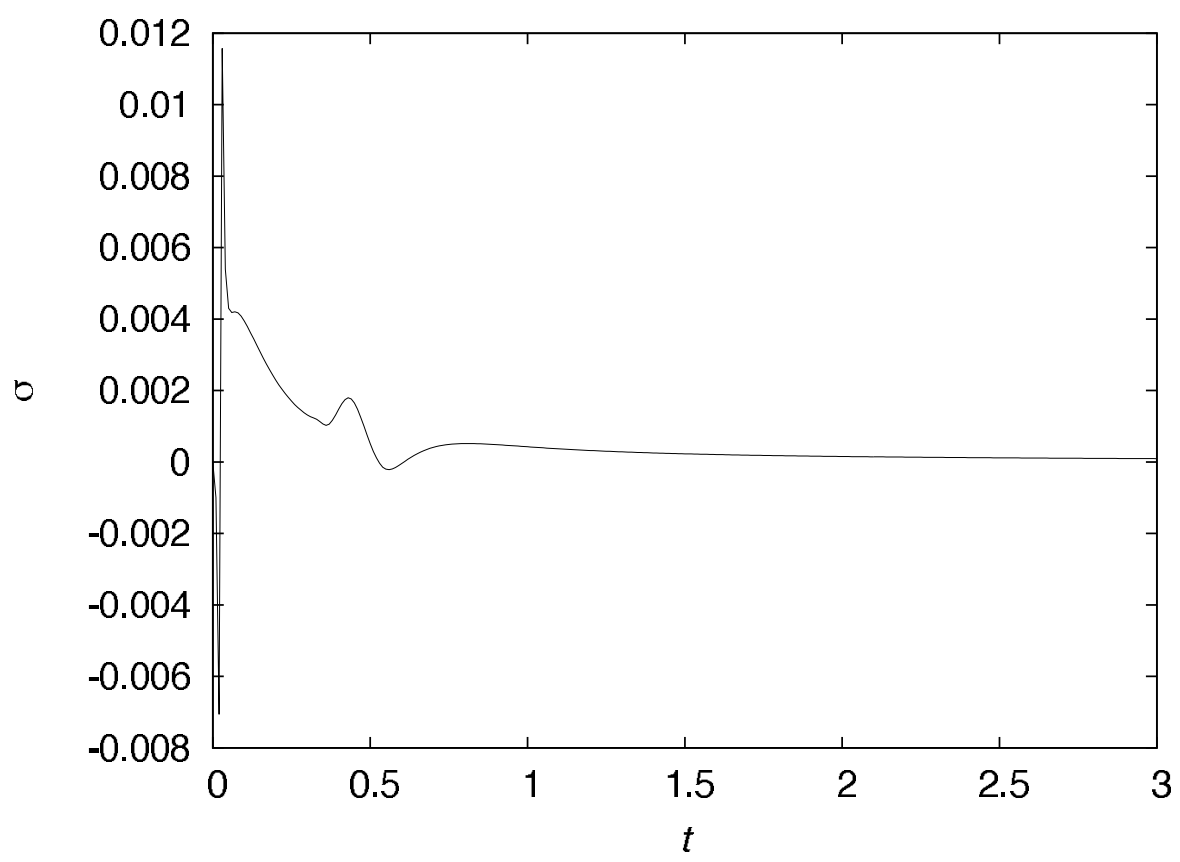

FiguRE 10. Relative error along a trajectory for the hydrogen oxidation model based on the same one-dimensional SIM calculation as in Fig. 9. The initial condition in the reduced model was $z=0.35$, and the full model was started from the corresponding point on the computed SIM. 


\section{Discussion and conclusions}

This paper brings together two important traditions in model reduction, namely methods based on timescale separation, and lumping. In the traditional approach to lumping, we seek lumping functions such that the lumped model has a closed equation of motion. In contrast, here we choose a lumping function and then find an equation for its slow timescale evolution after computing a SIM parameterized by the lumped variable(s). Li, Rabitz, and coworkers have developed related approaches in which they use singular perturbation theory to develop lumping schemes [29-31]. Singular perturbation theory is the archetypal time-scale separation method. In straightforward applications of the theory, a small parameter is identified that allows one to explicitly separate time scales $[4,12,23,32,53]$. In the work of Li and Rabitz, identification of the small parameter is not required [31]. This is also the case in our method. Provided the lumping function is physically sensible, we can proceed to construct the SIM, and thence to generate the reduced model.

The connection between time-scale separation and lumping was actually recognized at a very early stage: Wei and Kuo pointed out in 1969 that lumping had the effect of discarding some of the eigenvectors of a linear system of rate equations [57]. An appropriate choice of lumping functions ought then to specifically discard the fast eigenvectors in a linear system, retaining the slow time scales. Extending these ideas to the nonlinear regime brings us to the methods of Li and Rabitz [29-31].

Over the years, lumping has been applied in a number of areas of science, including ecological modeling $[2,24]$, hydrocarbon combustion modeling $[40,52,56]$, and industrial process control [1]. The current contribution provides another approach to obtaining lumped equations for systems where the separation of time scales is sufficiently large that only behavior on the slow time scale(s) is of interest. This raises the problem of generating initial conditions for the reduced model, an important problem studied by Cox and Roberts $[6,42]$. In the event that the transient behavior is also important, the fast fibers can be computed using existing methods $[55,59,60]$.

While the methods described here open up a new degree of freedom with respect to the computation of slow invariant manifolds, namely the choice of the parameterizing variables of the manifold via the choice of a lumping function, this of course creates a new set of issues. As illustrated in Fig. 2, the SIM must cross isosurfaces of the lumping function transversely. There is at present no simple way to guarantee this and, besides, we want to leave modelers the freedom, insofar as it is possible, to choose lumping functions that are meaningful in their respective application domains. That being the case, we would like to have a diagnostic for bad choices of the lumping function. We claim that the iterative method contains its own diagnostic element in that, even with stabilization, it will fail to converge if the relevant manifold cannot be represented in the form chosen, either because the lumping function fails to satisfy the transversality requirement, or because a manifold of the given dimension fails to exist in the region of space where the computation is being carried out [49]. Because we are solving the invariance equation, iterative stabilization, as applied in Section 4, can only succeed if there is a solution of the invariance equation to be computed [45], i.e. if there exists an invariant manifold of dimension $d$ which is at least locally the graph of a function of $\mathbf{z}$. In particular, some anomalies that can arise in the application of Galerkin projections such as the Karhunen-Loève decomposition, including projected dynamics in which the stability of embedded invariant orbits is reversed [41], or projected dynamics that lacks the structural stability properties of the parent vector field [36], are impossible because only invariant manifolds can be fixed points of an iterative process derived directly from the invariance equation. Moreover, the dynamics on the manifold is automatically retained by using the chain rule to obtain the evolution equations of the reduced model, equation (2.2).

In the two models we studied here, we used slightly different computational methods, in one case applying chained iteration after eliminating a subset of the variables with the lumping relation, and in the other solving the invariance and lumping relations as a system of nonlinear equations at each step. In previous work on linear lumping in the linear metabolic pathway model, we solved the invariance equation by chained iteration without regard to the lumping function [37]. We then summed the appropriate components of the $\mathbf{s}$ vector to infer the value of $\mathbf{z}$ to which each point on the manifold corresponds. This 
resulted in non-uniform grids that evolved during the computation. This does work, but only for linear lumping functions. All of these variations highlight the fact that there are a number of ways to solve the combined invariance and lumping equations. A systematic study of the effects of different computational sequences on the stability and rate of convergence of the calculations would be useful.

In the calculations reported here, we have used uniform meshes. As mentioned above, we have also worked with non-uniform meshes generated as a byproduct of a particular way of structuring the computation [37]. Clearly, it would be more efficient to use more mesh points in regions where one or more of the $s_{i}(\mathbf{z})$ display large curvature, and fewer where $\mathbf{S}(\mathbf{z})$ is relatively flat. Thus it would be interesting to find out if a deliberately adaptive mesh has positive effects on the iterative stability of the computation. The small but tricky examples studied in this contribution may be good test cases for tackling this question.

Acknowledgements. MRR benefited greatly from conversations with attendees at the recent conference on model reduction held in honor of Alexander Gorban in Leicester. In particular, he wishes to thank Ilya Karlin and Katalin Hangos for their useful comments and suggestions. MRR also wishes to thank David Kaminski for a useful discussion regarding the visualization of the connection between lumping and the geometry of the manifold. This work was supported by the Natural Sciences and Engineering Research Council of Canada.

\section{References}

[1] M. Aoki. Some approximation methods for estimation and control of large scale systems. IEEE Trans. Automat. Control, AC-23 (1978), 173-182.

[2] P. Auger, R. Bravo de la Parra. Methods of aggregation of variables in population dynamics. C. R. Acad. Sci., Ser. III, 323 (2000), 665-674.

[3] F. Büchel, N. Rodriguez, N. Swainston, C. Wrzodek, T. Czauderna, R. Keller, F. Mittag, M. Schubert, M. Glont, M. Golebiewski, M. van Iersel, S. Keating, M. Rall, M. Wybrow, H. Hermjakob, M. Hucka, D. B. Kell, W. Müller, P. Mendes, A. Zell, C. Chaouiya, J. Saez-Rodriguez, F. Schreiber, C. Laibe, A. Dräger, N. Le Novère. Path2Models: Large-scale generation of computational models from biochemical pathway maps. BMC Syst. Biol., 7 (2013), 116.

[4] F. W. Chang, F. A. Howes. Nonlinear Singular Perturbation Phenomena: Theory and Application. Applied Mathematical Sciences. vol. 56. Springer, New York, 1984.

[5] G.-M. Côme. Radical reaction mechanisms. Mathematical theory. J. Phys. Chem., 81 (1977), 2560-2563.

[6] S. M. Cox, A. J. Roberts. Initial conditions for models of dynamical systems. Physica D, 85 (1995), $126-141$.

[7] P. G. Coxson, K. B. Bischoff. Lumping strategy. 1. Introductory techniques and applications of cluster analysis. Ind. Eng. Chem. Res., 26 (1987), 1239-1248.

[8] P. G. Coxson, K. B. Bischoff. Lumping strategy. 2. A system theoretic approach. Ind. Eng. Chem. Res., 26 (1987), 2151-2157.

[9] C. F. Curtiss, J. O. Hirschfelder. Integration of stiff equations. Proc. Natl. Acad. Sci. U.S.A., 38 (1952), $235-243$.

[10] M. J. Davis, R. T. Skodje. Geometric investigation of low-dimensional manifolds in systems approaching equilibrium. J. Chem. Phys., 111 (1999), 859-874.

[11] G. Farkas. Kinetic lumping schemes. Chem. Eng. Sci., 54 (1999), 3909-3915.

[12] N. Fenichel. Geometric singular perturbation theory for ordinary differential equations. J. Differential Equations, 31 (1979), 53-98.

[13] S. J. Fraser. The steady state and equilibrium approximations: A geometrical picture. J. Chem. Phys., 88 (1988), $4732-4738$.

[14] S. J. Fraser. Symbolic methods for invariant manifolds in chemical kinetics. Int. J. Quantum Chem., 106 (2006), 228-243.

[15] J.-M. Ginoux, B. Rossetto, L. O. Chua. Slow invariant manifolds as curvature of the flow of dynamical systems. Int. J. Bifurc. Chaos, 18 (2008), 3409-3430.

[16] A. N. Gorban, I. V. Karlin, V. B. Zmievskii, S. V. Dymova. Reduced description in the reaction kinetics. Physica A, 275 (2000), 361-379.

[17] A. N. Gorban, I. V. Karlin. Method of invariant manifolds and regularization of acoustic spectra. Transport Theory Stat. Phys., 23 (1994), 559-632.

[18] A. N. Gorban, I. V. Karlin. Method of invariant manifold for chemical kinetics. Chem. Eng. Sci., 58 (2003), $4751-4768$.

[19] A. N. Gorban, I. V. Karlin, A. Yu. Zinovyev. Constructive methods of invariant manifolds for kinetic problems. Phys. Rep., 396 (2004), 197-403.

[20] A. N. Gorban, I. V. Karlin, A. Yu. Zinovyev. Invariant grids for reaction kinetics. Physica A, 333 (2004), $106-154$.

[21] D. A. Goussis, M. Valorani. An efficient iterative algorithm for the approximation of the fast and slow dynamics of stiff systems. J. Comput. Phys., 214 (2006), 316-346.

[22] H. Huang, M. Fairweather, J. F. Griffiths, A. S. Tomlin, R. B. Brad. A systematic lumping approach for the reduction of comprehensive kinetic models. Proc. Combust. Inst., 30 (2005), 1309-1316. 
[23] F. G. Heineken, H. M. Tsuchiya, R. Aris. On the mathematical status of the pseudo-steady state hypothesis of biochemical kinetics. Math. Biosci., 1 (1967), 95-113.

[24] Y. Iwasa, V. Andreasen, S. Levin. Aggregation in model ecosystems. I. Perfect aggregation. Ecol. Modelling, 37 (1987), 287-302.

[25] J. G. Kemeny, J. L. Snell. Finite Markov Chains. Springer, New York, 1976, pp. 123-140.

[26] J. C. W. Kuo, J. Wei. A lumping analysis in monomolecular reaction systems. Analysis of approximately lumpable systems. Ind. Eng. Chem. Fundam., 8 (1969), 124-133.

[27] S. H. Lam. Using CSP to understand complex chemical kinetics. Combust. Sci. Technol., 89 (1993), $375-404$.

[28] S. H. Lam, D. A. Goussis. The CSP method for simplifying kinetics. Int. J. Chem. Kinet., 26 (1994), 461-486.

[29] G. Li, H. Rabitz. Combined symbolic and numerical approach to constrained nonlinear lumping-with application to an $\mathrm{H}_{2} / \mathrm{O}_{2}$ oxidation model. Chem. Eng. Sci., 51 (1996), 4801-4816.

[30] G. Li, H. Rabitz, J. Tóth. A general analysis of exact nonlinear lumping in chemical kinetics. Chem. Eng. Sci., 49 (1994), 343-361.

[31] G. Li, A. S. Tomlin, H. Rabitz, J. Tóth. Determination of approximate lumping schemes by a singular perturbation method. J. Chem. Phys., 99 (1993), 3562-3574.

[32] C. C. Lin, L. A. Segel. Mathematics Applied to Deterministic Problems in the Natural Sciences. Classics in Applied Mathematics, vol. 1 SIAM, Philadelphia, 1988, ch. 9-10.

[33] Ch. Lubich, K. Nipp, D. Stoffer. Runge-Kutta solutions of stiff differential equations near stationary points. SIAM J. Numer. Anal., 32 (1995), 1296-1307.

[34] U. Maas, A. S. Tomlin. Time-scale splitting-based mechanism reduction. Cleaner Combustion (F. Battin-Leclerc et al., ed.), Springer, London, 2013, pp. 467-484.

[35] A. H. Nguyen, S. J. Fraser. Geometrical picture of reaction in enzyme kinetics. J. Chem. Phys., 91 (1989), $186-193$.

[36] B. R. Noack, K. Afanasiev, M. Morzynski, G. Tadmor, F. Thiele. A hierarchy of low-dimensional models for the transient and post-transient cylinder wake. J. Fluid Mech., 497 (2003), 335-363.

[37] B. E. Okeke. Lumping Methods for Model Reduction. Master's thesis, University of Lethbridge, 2013. URL: https: //www.uleth.ca/dspace/handle/10133/3558

[38] M. S. Okino, M. L. Mavrovouniotis. Simplification of mathematical models of chemical reaction systems. Chem. Rev., 98 (1998), 391-408.

[39] D. J. M. Park. The hierarchical structure of metabolic networks and the construction of efficient metabolic simulators. J. Theor. Biol., 46 (1974), 31-74.

[40] N. Peters, B. Rogg. Reduced Kinetic Mechanism for Applications in Combustion Systems. 2nd ed., Springer, Berlin, 1993.

[41] D. Rempfer. On low-dimensional Galerkin models for fluid flow. Theor. Comput. Fluid Dyn., 14 (2000), 75-88.

[42] A. J. Roberts. Appropriate initial conditions for asymptotic descriptions of the long term evolution of dynamical systems. J. Austral. Math. Soc. B, 31 (1989), 48-75.

[43] B. Rossetto, T. Lenzini, S. Ramdani, G. Suchey. Slow-fast autonomous dynamical systems. Int. J. Bifurc. Chaos, 8 (1998), 2135-2145.

[44] M. R. Roussel. A Rigorous Approach to Steady-State Kinetics Applied to Simple Enzyme Mechanisms. Ph.D. thesis, University of Toronto, 1994.

[45] M. R. Roussel. Forced-convergence iterative schemes for the approximation of invariant manifolds. J. Math. Chem., 21 (1997), 385-393.

[46] M. R. Roussel. Approximating state-space manifolds which attract solutions of systems of delay-differential equations. J. Chem. Phys., 109 (1998), 8154-8160.

[47] M. R. Roussel, S. J. Fraser. Geometry of the steady-state approximation: Perturbation and accelerated convergence methods. J. Chem. Phys., 93 (1990), 1072-1081.

[48] M. R. Roussel, S. J. Fraser. On the geometry of transient relaxation. J. Chem. Phys., 94 (1991), $7106-7113$.

[49] M. R. Roussel, S. J. Fraser. Global analysis of enzyme inhibition kinetics. J. Phys. Chem., 97 (1993), $8316-8327$. Errata, ibid. 98 (1994), 5174.

[50] M. R. Roussel, S. J. Fraser. Invariant manifold methods for metabolic model reduction. Chaos, 11 (2001), $196-206$.

[51] D. Shear. An analog of the Boltzmann H-theorem (a Liapunov function) for systems of coupled chemical reactions. J. Theor. Biol., 16 (1967), 212-228.

[52] A. Stagni, A. Cuoci, A. Frassoldati, T. Faravelli, E. Ranzi. Lumping and reduction of detailed kinetic schemes: an effective coupling. Ind. Eng. Chem. Res., 53 (2014), 9004-9016.

[53] A. N. Tikhonov, A. B. Vasil'eva, A. G. Sveshnikov. Differential Equations. Springer, Berlin, 1985, pp. 186-213.

[54] A. S. Tomlin, T. Turányi, M. J. Pilling. Mathematical tools for the construction, investigation and reduction of combustion mechanisms. Compr. Chem. Kinet., 35 (1997), 293-437.

[55] K. Uldall Kristiansen, M. Brøns, J. Starke. An iterative method for the approximation of fibers in slow-fast systems. SIAM J. Appl. Dyn. Syst., 13 (2014), 861-900.

[56] J. Warnatz, U. Maas, R.W. Dibble. Combustion: Physical and Chemical Fundamentals, Modeling and Simulation, Experiments, Pollutant Formation, 2nd ed., Springer, Berlin, 1999.

[57] J. Wei, J. C. W. Kuo. A lumping analysis in monomolecular reaction systems. Analysis of the exactly lumpable system. Ind. Eng. Chem. Fundam., 8 (1969), 114-123.

[58] L. E. Whitehouse, A. S. Tomlin, M. J. Pilling. Systematic reduction of complex tropospheric chemical mechanisms, Part I: Sensitivity and time-scale analyses. Atmos. Chem. Phys., 4 (2004), 2025-2056. 
[59] A. Zagaris, H. G. Kaper, T. J. Kaper. Analysis of the computational singular perturbation reduction method for chemical kinetics. J. Nonlinear Sci., 14 (2004), 59-91.

[60] A. Zagaris, H. G. Kaper, T. J. Kaper, Fast and slow dynamics for the computational singular perturbation method. Multiscale Model. Simul., 2 (2004), 613-638. 